\title{
Radiation Hydrodynamics in Solar Flares
}

\author{
George H. Fisher \\ Institute of Geophysics and Planetary Physics \\ Mail Code L-413 \\ Lawrence Livermore National Laboratory \\ Livermore, California 94550 \\ U.S.A.
}

\begin{abstract}
Solar flares are currently understood as the explosive release of energy stored in the form of stressed magnetic fields. In many cases, the released energy seems to take the form of large numbers of electrons accelerated to high energies (the nonthermal electron "thick target" model), or alternatively plasma heated to very high temperatures behind a rapidly moving conduction front (the "thermal" mode1). The transport of this energy into the remaining portion of the atmosphere results in violent mass motion and strong emission across the electromagnetic spectrum. Radiation processes play a crucial role in determining the ensuing plasma motion.
\end{abstract}

One important phenomenon observed during flares is the appearance in coronal magnetic loops of large amounts of upflowing, soft $x$-ray emitting plasma at temperatures of $1-2 \times 10^{7}[\mathrm{~K}]$. It is believed that this is due to chromospheric evaporation, the process of heating cool ( $\sim 10^{4}[\mathrm{~K}]$ ) chromospheric material beyond its ability to radiate. Detailed calculations of thick target heating show that if nonthermal electrons heat the chromosphere directly, then the evaporation process can result in explosive upward motion of $X$-ray emitting plasma if the heating rate exceeds a threshold value. In such a case, upflow velocities approach an upper limit of roughly $2.35 \mathrm{c}_{\mathrm{s}}$ as the heating rate is increased beyond the threshold, where $c_{s}$ is the sound speed in the evaporated plasma. This is known as explosive evaporation. If the flare heating rate is less than the threshold, evaporation takes place indirectly through thermal conduction of heat deposited in the corona by the energetic electrons. Upflows in this case are roughly 10 to $20 \%$ of the upper limit. Evaporation by thermal model heating always takes place through thermal conduction, and the computed upflow speeds seem to be about $10 \%$ to $20 \%$ of the upper limit, independent of the energy flux.

The pressure increase in the evaporated plasma for either the thick target or thermal model leads to a number of interesting phenomena in the flare chromosphere. The sudden pressure increase initiates a downward moving "chromospheric condensation", an overdense region which gradually decelerates as it accretes material and propagates into the gravitationally stratified chromosphere. Solutions to an equation of motion for this condensation shows that its motion decays after about one minute of propagation into the chromosphere. When the front of this downflowing region is supersonic relative to the atmosphere ahead of it, a radiating shock will form. If the downflow is rapid enough, the shock strength should be sufficient to excite UV radiation normally associated with the transition region, and furthermore, the radiating shock will be brighter than the transition region. These results lead to a number of observationally testable relationships between the optical and ultraviolet spectra from the condensation and radiating shock. 


\section{Introduction}

Solar flares are rather violent and extremely complicated phenomena, and it should be made clear at the outset that a physically complete picture describing all aspects of flares does not exist. From the wealth of data which is available, it is apparent that many different types of physical processes are involved during flares: energetic particle acceleration, rapid magnetohydrodynamic motion of complex field structures, magnetic reconnection, violent mass motion along magnetic field lines, and the heating of plasma to tens of millions of degrees, to name a few. The goal of this paper will be to explore just one aspect of solar flares, namely the interaction of hydrodynamics and radiation processes in fluid being rapidly heated along closed magnetic field lines. The models discussed are therefore necessarily restrictive, and will address only a few of the observed or observable phenomena.

In Section II, the structure of the solar corona is discussed, and evidence arguing for the use of the one dimensional loop model is presented. In Section III, two popular models for energy release in flares are presented, along with some brief discussion of pertinent observations. Section IV describes the physics included in the numerical simulations of radiative hydrodynamic response of flare loops to energy release. Specific results of such simulations and related analytical calculations are given in Sections V, VI, and VII, on the phenomena of chromospheric evaporation, the formation and propagation of very dense chromospheric condensations, and emission from radiating shocks, respectively. Finally, new results concerning possibly observable features of chromospheric condensations and radiating shocks are summarized in the final section, VIII.

\section{Solar A tmospheric Structure: The Loop Model}

One of the most important discoveries to come out of space based observations of the sun's corona is the degree to which the structure of the upper solar atmosphere is determined by magnetic fields. In the absence of magnetic fields, a balance between gravity and gas pressure would result in a spherically symmetric distribution of matter. However, spatially resolved observations of the sun's coronal X-ray emission show this is far from the case [1]. There are large patches of the sun's surface which appear to show essentially no X-ray emission. These are called coronal holes, which are believed to be regions where the magnetic field lines from the surface open into interplanetary space. Gas pressure gradients along these diverging field lines are believed to drive the solar wind against the opposing gravitational force. As a result of its rapid expansion, the coronal material has a low density, and it therefore emits few $X-$ rays. Most of the solar surface, however, appears to covered by closed magnetic field lines: A single magnetic field line connects two different points of the sun's surface, denoted as magnetic footpoints. Coronal X-ray emission from the magnetically closed region seems to consist of emission from individual flux tubes, or bundles of adjacent field lines, each with its own characteristic brightness. The brightest and most visible flux tubes occur in active regions, where the photospheric magnetic field tends to be both strong and complex. The difference in observed brightness between individual flux tubes can be orders of magnitude, implying that a large range in coronal density also exists between individual coronal flux tubes, or coronal loops as they are of ten called. This leads one to conclude that for the purposes of describing the $\mathrm{X}$-ray emitting corona, the coronal loop can be considered as a basic unit of structure. Furthermore, one can take advantage of the wide range in inferred loop densities to note that the magnetic forces must be strongly dominant over those from gas pressure gradients: two different coronal loops of the same height would have roughly the same gas pressure if magnetic forces were weak or comparable to pressure gradients and gravity. Thus pressure gradients tend to drive mass motion only along the direction of the field lines. 
In addition to being the most important agent in determining the structure of the upper solar atmosphere, magnetic fields are also believed to be the energy source for many dynamic solar phenomena, including, but not restricted to, solar flares. It is generally believed that flares occur when stresses in the magnetic fields which accumulate through motion of the magnetic footpoints (by differential rotation of the sun's surface, for example) result in rapid and explosive reconfiguration of the field lines into a lower energy state. The energy that is released from this explosive phenomenon takes a wide variety of forms, including the escape into space of energetic particles, violent mass motion along coronal flux tubes, and strong emission across the electromagnetic spectrum from radio waves to $\gamma$-rays.

The morphology of solar flares is in general very complex, and it is of ten difficult to make meaningful generalizations. Nevertheless, solar flares are often divided into two types depending on their geometric structure [2]. The compact flare apparently takes place in one or a few small closed coronal loops which do not seem to alter their size or shape during the flare, but which do become very bright in soft $x$-rays as the flare progresses. This implies that over the course of the flare, the magnetic pressure always dominates over the rapidly increasing gas pressure, as the loop would otherwise be blown apart as it filled up with hot plasma. Another type of flare commonly seen is called the two ribbon flare, which seems to consist of a wide arcade of bright coronal loops, the chromospheric footpoints of which form two bands or ribbons when viewed in visible emission such as $H \alpha$. The coronal loop arcade and the Ha ribbons combine to form a structure much like a covered wagon. The $\mathrm{H} \alpha$ ribbons are often observed to brighten and move apart over the course of the flare. [2] Two ribbon flares also seem to be associated with some open field lines, [3] and some models propose that the expansion of the Ha ribbons can be understood in terms of reconnection of open field lines into closed loop structures. [4]

Because of its apparently much simpler nature, most efforts at modeling the radiative hydrodynamic response to explosive flare energy release have focused on the compact flare picture: Flare energy is released within a single closed coronal loop model, whose size and shape are not allowed to change over the course of a calculation. Mass motion is allowed to occur only along the direction of the magnetic field, owing to the inability of the transverse pressure gradient to push the field lines out. In the following section, aspects of observations which have bearing on models for flare heating and energy transport are reviewed, along with two different pictures for how the flare energy is released.

\section{Flare Observations and Energy Release Models}

Studies of radiated emission indicate that flares occur in two different stages. First, during the relatively short impulsive phase, emission seen in hard $X$-rays (meaning photon energies greater than $20[\mathrm{KeV}]$ ) consists of many individual spikes ranging in duration from tens of milliseconds to tens of seconds. Impulsive phase emission is also seen in UV lines such as OV [5], optical line emission such as Ha [6], and frequency integrated EUV emission in the 10 to 1030 [月] range [7]. The impulsive phase is followed by the longer gradual or thermal phase, which is characterized by bright $X$-ray emission from an optically thin thermal plasma with temperatures of roughly $1-2 \times 10^{7}[\mathrm{~K}]$, gradually decaying over time scales of 20 minutes to one hour. It has been recently shown by observations from the Solar Maximum Mission (SMM) that blue shifts in CaXIX X-ray line emission indicating significant upflows are present during the impulsive phase. Upflows seem to begin and end simultaneously with hard $x$-rays. During this same period of time, the thermal soft $X$-ray emission measure is rapidly increasing, and reaches its peak at about the same time as cessation of hard $x$-ray emission. [8] The temporal relationship between impulsive phase emission, blue shifts, and the soft $X$-ray emission measure suggests that there is some causal connection between them. In the context of the simple compact loop flare, two models of how the flare energy is released into the atmosphere have been proposed. 
The thick target model $[9,10]$ assumes that all of the impulsive phase flare energy is released (in an unspecified manner) into the acceleration or energization of energetic nonthermal electrons, which then bombard the atmosphere along the coronal loop. Collisions of the energetic electrons with ions in the atmosphere then produce the observed hard $X$-rays by nonthermal bremstrahlung, and collisions with the ambient electrons produce heating. The upper chromosphere, in this scenario, is heated sufficiently to become thermally unstable, and then heats up rapidly to coronal temperatures. This "evaporated" material then expands upward into the coronal loop, giving rise both to the observed blue shifted emission seen in CaXIX, as well as the rapid rise in the total soft $X-r a y$ emission measure. In the meantime, the electrons penetrating further down into the chromosphere heat it as well, but below the threshold needed for evaporation. This portion of the chromosphere quickly reaches quasi-steady equilibrium between particle heating and radiative losses. The resulting increase in radiative losses is then alleged to account for the impulsive component of the optical, UV, and EUV emission.

The thermal model, on the other hand, assumes that the energy release takes place by heating some coronal plasma to very high temperatures (approximately $10^{8}$ [K]), and that this energy is then transported to the rest of the flare loop through the propagation of a thermal conduction front. During the initial stage, before the conduction front has had a chance to reach the chromosphere, the hot thermal plasma behind the front is alleged to account for the impulsive phase hard $X$-rays through the emission of thermal bremstrahlung. When the conduction front reaches the chromosphere, it begins the process of evaporation, and once again the evaporating material is alleged to account for both the observed blue shifts of CaXIX and the increase in the soft X-ray emission measure. The emission of UV, optical, and EUV radiation in the thermal model occurs either through the escape of a small fraction of the most energetic electrons through the conduction front into the chromosphere, or through heating of the chromosphere when the front impacts it.

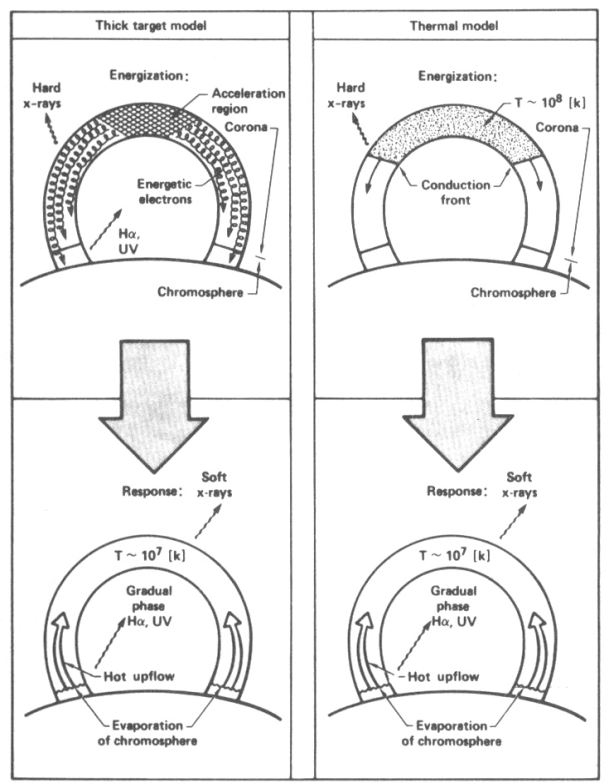

Figure 1. Schematic diagram showing how loop is energized in the thick target and thermal model scenarios. Note response is alleged to be same for both models

Both the thick target and thermal models, which are illustrated schematically in Fig. 1, represent extreme and idealized assumptions about how the impulsive phase flare energy is released. In the thick target case, the flare energy is 
released over a wide range of column depth, while the energy release in the thermal model is localized to the narrow column depth region of the preflare corona. Nevertheless, these two models are useful conceptually, as certain aspects of the observations are easily understood in terms of them. In addition, the thick target and thermal models produce heating functions which can easily be computed when doing radiation-hydrodynamic modeling of the response of a compact loop structure to impulsive phase heating. The goal of doing such modeling is to investigate in detail just exactly what the response of a model loop is to each form of flare heating, to discover which aspects of the models agree quantitatively with observations, and to investigate differences in the computed response which might suggest observational tests for discriminating between the two heating models.

IV. Numerical Modeling of Flaring Loops

\section{A. The Equations}

It is clear that the hydrodynamic and radiative response of a model loop atmosphere to flare heating is a complicated, non-linear problem. Therefore, much effort has been devoted to numerical solutions of both the hydrodynamic response. of the flare loop [11-20] as well as calculations of the radiative output of semiempirical flare models $[21,22]$. It is clear, however, that the most complete approach is the self-consistent solution of both the equations of hydrodynamics and radiation transport in the flaring loop. The first successful treatment of radiation-hydrodynamics in flare loops was developed by MCCLYMONT and CANFIELD [23]. These methods have since been used to study nonlocal radiation transport effects in quiet loop models (CANFIELD, FISHER, and MCCLYMONT [24]), the 1inear stability of quiet loop models (MCCLYMONT and CANFIELD [25]), the nonlinear evolution of unstable loop models (AN, CANFIELD, FISHER, and MCCLYMONT [26]), and the response of loops to both thick target (FISHER, CANFIELD, and MCCLYMONT $[27,28,29]$ ) and thermal (EISHER [30]) models of flare heating. The details of the physics included in the modeling of flaring loops, as well as some brief discussion of the numerical techniques, will be described in this section.

The compact loop is assumed to be semi-toroidal, with footpoints at either end imbedied in the photosphere. The loop is also assumed to be symmetric about the apex, so that only half the loop is actually modeled. The initial loop structure contains a corona, transition region, chromosphere, and the upper portion of the photosphere, all in hydrostatic and energetic equilibrium, as described by [26]. The cross sectional area of the loop is assumed not to vary along its length. The model loop geometry is shown schematically in Fig. 2. The equation of motion for the fluid within the loop is

$$
\mathrm{m} \partial \mathrm{v} / \partial \mathrm{t}=-\partial \mathrm{P} / \partial \mathrm{N}+\mathrm{mg} / /+\partial / \partial \mathrm{N}[(4 / 3) n \eta \partial \mathrm{v} / \partial \mathrm{N}]
$$

where the independent spatial variable $N$ (the column depth) is given by

$$
\mathrm{N}=\int_{0}^{\mathrm{z}} \mathrm{nd} \mathrm{z}^{\prime},
$$

measured from the loop apex. The quantity $n$ is the density of equivalent hydrogen atoms (i.e. $n=n_{H}+n_{p}$ ). The quantity $m$ is the mean mass per hydrogen nucleus in the solar atmosphere.

The continuity equation is written, for computational purposes as the pair of equations

$$
n=(\partial z / \partial N)^{-1} ; \partial z / \partial t=v,
$$

from which the conventional form is easily obtained.

The energy equation is written

$$
\partial \varepsilon / \partial t=Q_{a}+Q_{f l}-R+C-P \partial v / \partial N+(4 / 3) n \eta|\partial v / \partial N|^{2}
$$


where the quantity $\varepsilon$, the internal energy per equivalent $H$-atom, is given by

$$
\varepsilon=3 / 2(1+y+x) k T+\sum_{\ell} \varepsilon_{\ell} \varphi_{l}
$$

where $\varepsilon_{\ell}$ is the energy of a hydrogen atom in quantum state $\ell$, measured from the ground state, $\phi_{\ell}$ is the fractional population of state $\ell\left(\phi_{\ell} \equiv n_{\ell} / n\right)$, $y$ is the number of nonhydrogenic atoms per $H$-atom, and $x$, the ionized fraction, is the number of free electrons per $\mathrm{H}$-atom. The quantity $Q_{a}$ here is the quiescent energy needed to keep the preflare loop in energetic equilibrium, $Q_{f l}$ is the flare heating function, $R$ is the contribution to radiative losses (or radiative heating), and $C$ is the contribution to heating or cooling from thermal conduction. These quantities will be described in more detail in the next subsection. The fifth term in (4) is just the compression term, and the last term in both (1) and (4) includes the effects of viscosity; the viscosity $n$ contains both a physical component and a pseudoviscosity component used for handling shocks.

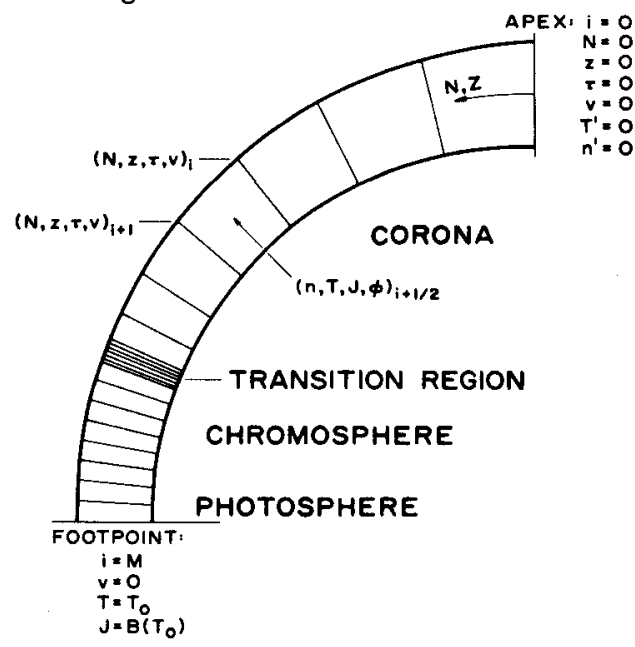

Figure 2. Schematic diagram showing numerical model of symmetric flare loop

In addition to the hydrodynamic equations, the radiation transport and atomic rate equations for a two-level-plus-continuum hydrogen atom are also solved. This is necessary in order to self-consistently determine the ionized fraction $x$ and the optically thick hydrogenic losses in the chromosphere. The radiation transport equation is reduced to the probabilistic form [31],

$$
\mathrm{d} / \mathrm{d} \tau_{\mathrm{ul}}\left(\mathrm{J}_{\mathrm{ul}}-\mathrm{S}_{\mathrm{ul}}\right)=-2\left(\mathrm{p}_{\mathrm{u \ell}}\right)^{1 / 2} \mathrm{~d} / \mathrm{d} \tau_{\mathrm{u} \ell}\left(\mathrm{p}_{\mathrm{u}}{ }^{1 / 2} \mathrm{~S}_{\mathrm{u \ell}}\right)
$$

where the optical depth $\tau_{u l}$ is related to the atomic photoabsorption cross section $\sigma_{u \ell}$ by

$$
\mathrm{d} \tau_{\mathrm{u} \ell^{\prime}} \mathrm{dN}=\sigma_{\mathrm{ul}} \text {. }
$$

The quantities $J_{u \ell}$ and $S_{u l}$ represent the mean intensity and source function in transition ul, respectively, and $p_{u l}$ is the single flight escape probability for a photon at optical depth ${ }^{\top} u l$. The atomic rate equations are

$$
\partial \phi_{1} / \partial t=\sum_{j \neq i}\left(R_{j i} \phi_{j}-R_{i j} \phi_{i}\right),
$$

where $R_{i j}$ is the total transition rate from state $i$ to state $j$, including both collisional and radiative contributions.

The numerical methods used to solve the system of equations (1) through (8) are described in [23] and [27]. Briefly, the partial differential equations 
are converted to second order accurate finite differences in column depth and time. Because of the huge range in column depth (and spatial) scales in the problem (length scales between the transition region and corona can change by 4 orders of magnitude or more!) a uniform grid is not possible. Therefore a nonuniform, dynamically adjusted grid is essential in order that steep features remain numerically resolved. At the end of each time step, the numerical resolution is checked, and zones are split and merged as needed. Since the finite difference equations are centered in time, they must be solved implicitly. A generalized Newton-Raphson technique is used to solve the difference equations at each time step.

\section{B. Flare Heating, Radiative Losses, and Thermal Conduction}

The quantity $Q_{f l}$ in the energy equation represents flare heating within the model loop. The specific form of $Q_{f l}$ depends on the heating model chosen. In the case of thick target heating it is assumed that the energetic electrons are injected near the loop apex. For a specified input spectrum of electrons (power law $\delta$ and low energy cutoff $E_{c}$ ) the heating rate depends only on the column depth $N$, and has the following form [27]:

$$
Q_{t t}(N)=\left[(\delta-2) / 6 N_{c}\right] B(\delta / 2,1 / 3) \alpha^{-\delta / 2}\left\{a-b\left[N /\left(\alpha N_{c}\right)\right]^{2}\right\} F(t),
$$

for $N \leq \alpha N_{c}$, and

$$
Q_{t t}(N)=\left[(\delta-2) / 6 N_{c}\right] B(\delta / 2,1 / 3)\left[N / N_{c}\right]^{-\delta / 2} F(t)
$$

for $N \geq a N_{c}$. The quantity $N_{c}$, the stopping depth of electrons with cutoff energy $E_{c}[\mathrm{KeV}]$, is given by $\mathrm{N}_{c}=E_{c}^{2} /\left(3 K_{\text {coll }}\right)$, where $K_{c o l l}=3.64 \times 10^{-18}$ $\left[\mathrm{KeV}^{2} \mathrm{~cm}^{2}\right]^{c}[9]$. The quantity $B(x, y), j$, the beta function, and $a=1+\delta / 4$; $b=\delta / 4$; and $\alpha=[(\delta / 9) \mathrm{aB}(\delta / 2,1 / 3)]^{2 /(\delta-2)}$. For all of the thick target simulations discussed here, the low energy cutoff $E_{c}$ was chosen to be 20 $[\mathrm{KeV}]$, and the power law index $\delta$ to be 4 . In the case of thermal model heating, the heating function is assumed to be gaussian in column depth about the loop apex:

$$
Q_{t h}(N)=2 /\left(\pi^{1 / 2} \sigma\right) e^{-(N / \sigma)^{2}} F(t)
$$

where the heating region $N \leq \sigma$ is in the preflare corona. In both cases, $F(t)$ is the total energy input rate [erg $\mathrm{cm}^{-2} \mathrm{~s}^{-1}$ ] into the loop as a function of time.

The radiative term " $R$ " in the energy equation is broken into a number of components. The contribution from hydrogen is computed from the solutions to the atomic rate and radiation transport equations (8) and (6); it is $g^{i}$ ven by

$$
R_{H}=\sum_{u l} h v_{u \ell}\left(R_{u \ell} \phi_{u}-R_{\ell u} \phi_{\ell}\right)
$$

where $u, l=1,2, c . \quad R_{1 j}$ is the sum of both collisional and radiative rates. More details are given in [23] and [27]. It is well known that CaII and MgII are important contributors to radiative losses in the chromosphere, but are also optically thick. Because of the large number of levels needed to properly describe these ions, it is not presently possible to include them in the radiation transport portion of the code. However, an approximate method of computing losses from these important ions was developed which was computationally expedient, yet accounted for the most important optical depth effects. This is described in the appendix of [27]. The radiative losses from the $\mathrm{H}^{-}$continuum are adopted from HENOUX and NAKAGAWA [32], who use an optically thin approximation. The radiative loss rate from the hydrogen freefree continuum is from RICCHIAZZI [33], and is also optically thin, but includes an incident photospheric radiation field. The contribution of all the other elemental species is assumed to be optically thin. This loss rate is taken from [34], but with the hydrogen, calcium, and magnesium 
contributions removed [35], since these are calculated explicitly in the code.

The term " $C$ " in the energy equation is the contribution to heating or cooling by thermal conduction, and is given by $\mathrm{C}=-\mathrm{dF}_{\mathrm{c}} / \mathrm{dN}$, where $\mathrm{F}_{\mathrm{c}}$ is the conductive flux. Typically, the conductive flux is assumed to be given by [36]

$$
F_{c l}=-K\left(T, n_{e}\right) d T / d z \text {. }
$$

However, this expression for the conductive flux breaks down if the temperature gradient becomes too steep: the flux cannot exceed that given by all the local electrons moving in one direction at their thermal speed. This defines an upper bound to the conductive flux

$$
\left|F_{\text {sat }}\right|=\operatorname{an}_{e^{T^{3 / 2}}}
$$

where the quantity a is related to the experimentally derived "flux limiter" f from laser ablation experiments as

$$
a=\left(k_{b}^{3 / m_{e}}\right)^{1 / 2} \mathbf{f} \text {. }
$$

Compatibility with laser ablation studies suggests that $f$ is roughly 0.1 [37]. As an Ansatz then, the conductive flux $\mathrm{F}_{\mathrm{c}}$ is taken to be

$$
\mathrm{F}_{\mathrm{c}}=\mathrm{F}_{\mathrm{cl}} /\left(1+\mathrm{F}_{\mathrm{cl}} / \mathrm{F}_{\mathrm{sat}}\right) \text {, }
$$

from which $\mathrm{C}=-\mathrm{dF} \mathrm{c}^{\prime} \mathrm{dN}$ is computed [27]. A shortcoming of these calculations is that heat conduction becomes an intrinsically non-local problem when the electron mean free path exceeds the temperature scale height (as can easily happen during a flare), and the contribution to local conductive heating depends globally on the temperature distribution. No attempt to include such effects has been included in this code. Recent progress has been made, however $[38,39]$, which may allow the inclusion of nonlocal conduction effects into radiation hydrodynamic codes in the future.

\section{Overview of the Numerical Simulations}

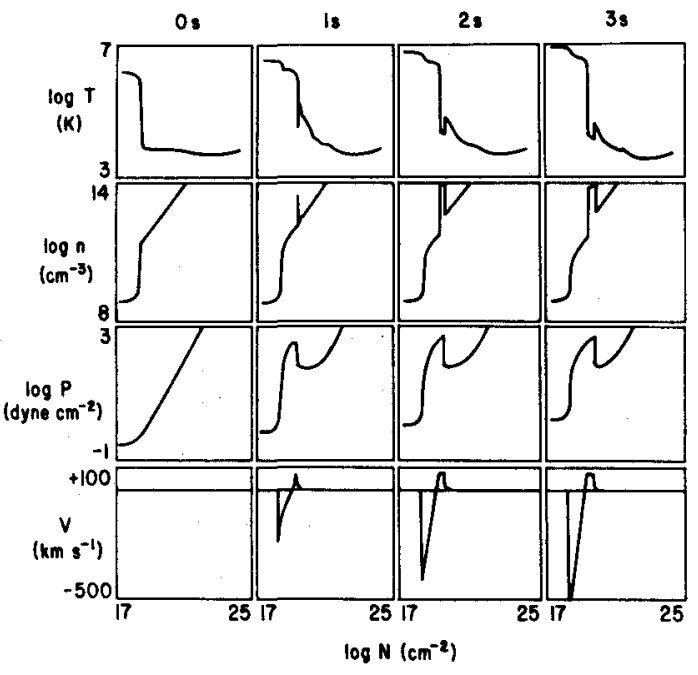

Figure 3. Evolution of temperature, number density, pressure, and velocity in the loop atmosphere for a thick target energy flux of $10^{11}\left[\mathrm{erg} \mathrm{cm} \mathrm{s}^{-2}\right]$. The column depth $\mathrm{N}$ is measured from the loop apex. Note velocities away from the loop apex (i.e. downward) are considered positive 
In this subsection, the overall results of the numerical simulations are discussed in order to impart some feeling for the most important physical mechanisms during the impulsive phase. Turning first to the thick target model, the simulation shown in Fig. (3), one notices a number of important phenomena. First of all, shortly after the onset of electron heating at $t=0$ [s], the topmost portion of the chromosphere heats up very rapidly (on a time scale of about 1 second) to coronal temperatures. This seems to happen more or less at constant density, resulting in a tremendous overpressure in this region. This overpressure quickly begins to drive upward motion of the heated material at speeds in excess of several hundred $\left[\mathrm{km} \mathrm{s}^{-1}\right]$. At the same time, the overpressure also begins to drive downflows into into the remaining portion of the chromosphere, but at speeds generally less than $100\left[\mathrm{~km} \mathrm{~s}^{-1}\right]$. Interestingly, this downflowing region is both much denser and much cooler than the chromospheric material ahead of it. The effects of heating by nonthermal electrons below this downward moving "chromospheric condensation" seem mainly to be the heating of the chromosphere sufficiently to ionize it, and to maintain a quasi-steady balance between heating by the nonthermal electrons and the dominant radiative loss mechanism, which in this case is optically thin metal losses.
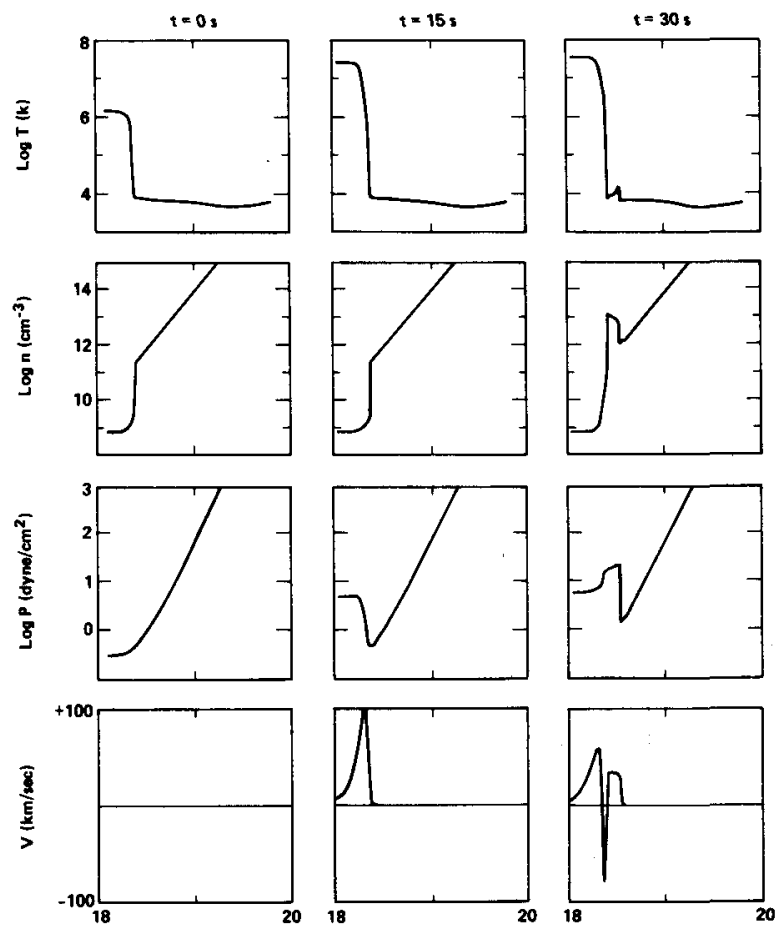

$\log N\left(\mathrm{~cm}^{-2}\right)$
Figure 4. Evolution of temperature, number density, pressure, and velocity in the loop atmosphere for thermal model heating with an energy input rate of $10^{9}$ [erg cm $\left.\mathrm{s}^{-1}\right]$. The column depth $\mathrm{N}$ is measured from the loop apex. Note velocities away from the loop apex (i.e. downward) are considered positive

In the thermal model of flare heating, illustrated in Fig. (4), the evolution is somewhat different. After heating commences at $t=0$ [ $s]$, the upper portion of the corona corresponding to the energy deposition region rapidly achieves a temperature of roughly $3 \times 10^{7}[\mathrm{~K}]$, after which the additional flare heating goes into driving a saturated conduction front into the remaining portion of the ambient corona. It takes nearly 25 [s] for the conduction front to traverse the $30000[\mathrm{~km}]$ coronal portion of the loop before finally reaching the chromosphere. During this time, the coronal plasma is actually moving downward. When the front hits the top of the chromosphere, it begins to rapidly heat the dense material to temperatures of roughly $10^{7}[\mathrm{~K}]$, resulting in a local overpressure, although not as great as that for thick target explosive evaporation. This overpressure then drives upward motion of 
the heated material, turning around the earlier downward motion of coronal plasma. The upflow speeds, however, are not as great as in the thick target case, being just over $100\left[\mathrm{~km} \mathrm{~s}^{-1}\right]$. The overpressure also drives downflow into the chromosphere, as with the thick target case, but the temperatures involved are much lower, since there is not any direct heating source in the chromosphere. The amplitude of the downflow speed is also less, a result of the reduced overpressure. The dominant radiative loss mechanisms in the chromosphere for thermal model heating seem to be optically thick emission from hydrogen and singly ionized calcium and magnesium.

\section{Chromospheric Evaporation}

In the standard impulsive phase scenario, the observed rapid $r i s e$ in the soft $\mathrm{X}$-ray emission measure is attributed to chromospheric evaporation, the process of heating the upper chromosphere past the point of thermal instability; the temperature then quickly increases to roughly $10^{7}[\mathrm{~K}]$. As a result of this rapid heating, the evaporated plasma expands upward, producing the observed soft $X$-ray line blue shifts. One very interesting issue which the numerical simulations can address is the extent to which computed upflow speeds match the upflows inferred from observed blue shifts.

In discussing calculations of chromospheric evaporation and the ensuing upflows, it is useful to note that if impulsive phase flare heating takes place in a loop which is initially in hydrostatic equilibrium, then the upflow velocity is bounded above by $v_{*}[28,40]$, where

$$
v_{*}=\left[(6 / 5) \ln \left(n_{c h} / n_{c o}\right)\right]^{1 / 2} c_{s} \text {, }
$$

$\mathrm{n}_{\mathrm{ch}}$ and $\mathrm{n}_{\mathrm{co}}$ are the preflare values of the chromospheric and coronal densities, and $c_{s}$ is the adiabatic sound speed in the evaporated plasma. Using a "typical" value of $n_{c h} / n_{c o}=100$ gives $v_{*}=2.35 c_{s}$, a number valid over a surprisingly wide range of conditions because of the slow functional variation of $v_{*}$ with $n_{\mathrm{ch}^{\prime}} \mathrm{n}_{\mathrm{co}}$. The upper limit implies that the strength of any associated coronal shocks driven by the upflows are also limited [28]. The upper limit $v_{*}$ provides a normalized framework for discussing the upflow velocities obtained in many of the simulations of chromospheric evaporation done to date. When the quantity $v_{\max } / v_{*}$ is plotted against fiare heating rate (where $v_{\max }$ is the maximum upflow speed seen in a given simulation), an interesting trend is seen: At low energy input rates, both the thermal and thick target heating models yield maximum upflow speeds which are roughly 10 to $20 \%$ of $\mathbf{v}_{*}$. However, at high input rates, the thick target upflows are rapid and explosive, with maximum speeds of 70 to $100 \%$ of $v_{*}$. The thermal heating model, however, yields upflows at high heating rates which are only slightly greater than those at low heating rates.

It is fairly easy to understand the difference in response of the loop atmosphere to high and low heating fluxes for the thick target case. Roughly speaking, a sizeable fraction of the flare energy is deposited over a column depth $N_{c}$, the stopping depth of electrons at the low energy cutoff at $E_{c^{\circ}}$. Since $\mathrm{N}_{c}$ is generally large compared to transition region column depths in preflare loop models, a significant fraction of the energy is deposited directly in the chromosphere. At low energy fluxes, the chromospheric temperature simply rises until radiative losses balance the increase in the heating rate. Evaporation takes place when that portion of the flare energy deposited in the corona results in an increased conductive flux into the transition region, resulting in a compensating enthalpy flux in the opposite direction. This scenario has been labeled "gentle" evaporation. At high energy fluxes, however, the upper portion of the chromosphere is unable to radiate away the flare energy that is being deposited there. It therefore heats up en masse to coronal temperatures, and then expands rapidly into the overlying corona. This is labeled "explosive" evaporation. A consequence of understanding this mechanism is that one can calculate the "threshold" heating 
flux for explosive evaporation to occur by simply equating the flare heating rate at the top of the chromosphere with the peak radiative loss rate there. A simple analytic model has been developed [28] which reproduces the temporal upflow behavior in several published simulations of explosive evaporation. Basically, this "gasbag" model assumes that the evaporated material heats up isochorically until a maximum temperature is reached; the plasma is then assumed to expand isothermally in a homologous fashion.

Evaporation from thermal heating models is driven by thermal conduction of heat from the corona. Since the flare energy is being deposited in the corona, and radiative losses there are not terribly effective, the corona responds by heating up and driving a larger conductive flux into the transition region, leading to evaporation much like that of the "gentle" case for thick target heating, except that a much larger fraction of the released energy goes into the evaporation process.

The observations of blue shifts and coronal temperatures derived from soft $X$-ray lines seem to lend support to the thermal model, or to "gentle" evaporation in the thick target model, if one uses a smaller low energy cutof $f$ than is generally assumed. Inferred upflow velocities range from 10 to $30 \%$ of the upper limit $v_{*}[40]$, compatible with those seen in numerical simulations of thermal model heating or thick-target "gentle" evaporation. Information on the low energy cutoff is observationally inaccessible because of the difficulty in unfolding the hard $x$-ray spectrum from the thermal soft $x$-ray source below $20[\mathrm{KeV}]$.

\section{Formation and Evolution of Chromospheric Condensations}

One of the most striking features seen in Figures 3 and 4 showing the response of the loop atmosphere to impulsive phase flare heating is the formation in the chromosphere of downward moving, overdense regions. Furthermore, in the thick target case (Fig. 3) the downflowing dense plasma is also significantly cooler than its surroundings. These "chromospheric condensations" form shortly after the onset of chromospheric evaporation, and are in fact driven by the evaporation process. In the case of thick target evaporation (Fig. 3) the pressure in the evaporated region exceeds the overlying coronal pressure as well as the pressure in the chromosphere. This results in driving both upward motion (evaporation) and the downward moving chromospheric condensation. The hydrodynamic response for the thermal model (Fig. 4) is similar: The downward moving conduction front hits the top of the chromosphere, suddenly creating a local pressure maximum at that point. Again, this drives both upflows (evaporation) and downflows (the chromospheric condensation), as the conduction front propagates into the chromosphere.

The structure of the chromospheric condensation is as follows: If the condensation's velocity is supersonic relative to the material ahead of it, the leading edge of the condensation consists of a hydrodynamic shock, which is of order one proton-proton mean free path thick (or less if the shock is collisionless). Behind the shock is a region of rapid radiative cooling. In the thick target case, this region is very thin. The shock and cooling region combined together form a "radiating shock", a structure well known in the astrophysics of supernova remnants, for example. If the condensation front is moving subsonically, then there is no radiating shock, but there is still a front present, which is approximately one radiative cooling length thick. For thick target heating, this cooling length is so short that the front remains quite thin.

In the thick target case, the radiating shock (if it exists) is followed by a region which is in quasi-steady energetic equilibrium, in which nonthermal electron heating balances radiative losses. This region makes up nearly all the mass of the condensation, with the radiating shock forming only at the very front. In fact, for the purposes of describing the jump in conditions on 
either side of the radiating shock (i.e. from in front of the condensation to within it) the radiating shock itself can be completely ignored: In the moving frame of the shock front, the conservation of $\rho v$ and $P+\rho v^{2}$ across the front as well as the constraint that thick target heating (per unit mass) must balance radiative losses on either side of the radiating shock, unambiguously specifies what the jump in hydrodynamic variables must be across the radiating shock. (This same argument holds true whether the "condensation front" consists of a radiating shock or not). If the plasma is completely ionized on both sides of the front (which for thick target heating is true), and if the dominant radiative losses are optically thin (which they are for thick target heating) specified by a power law $\left(\Lambda(T)=a T^{\alpha}\right)$, the jump conditions across the condensation front can be written in the following form:

$$
\begin{gathered}
\left(v_{1} / v_{2}\right)=\left(n_{2} / n_{1}\right)=\left(T_{1} / T_{2}\right)^{\alpha}=p^{1 / \Gamma}, \\
M_{1}=v_{1} / c_{1}=\left\{\left[p^{-1}\right] /\left[\Gamma\left(1-p^{-1 / \Gamma}\right)\right]\right\}^{1 / 2}, \\
N=n_{1} v_{1}=n_{2} v_{2}=n_{1} c_{1} M_{1}, \\
v_{d}=v_{1}-v_{2}=M_{1} c_{1}\left(1-p^{-1 / \Gamma}\right),
\end{gathered}
$$

where $p=\left(p_{2} / p_{1}\right), \Gamma=(\alpha-1) / \alpha$, and $c_{1}=\left(\Gamma p_{1} / p_{1}\right)^{1 / 2}$ is the long wavelength limit of the sound speed in a plasma with optically thin losses balancing a heating rate constant per unit mass [29]. Subscript 1 refers to material ahead of the condensation front, and 2 to the material behind the front (within the condensation itself). The application of these simple jump conditions to the thick target condensation shown in Fig. (3) describes its instantaneous evolution quite well [29].

The chromospheric condensation associated with the thermal model shows some significant differences from the thick target condensation. In the latter case, the conditions within most of the condensation are determined by quasisteady energy balance, while the radiating shock forms only a thin layer at the front of the condensation. In the thermal model, there is no direct release of flare energy into the chromosphere. The unevaporated chromosphere remains much cooler overall, and hence has a much longer radiative cooling time. In this case, the cooling portion of the radiating shock encompasses virtually the entire condensation, i.e. there is essentially no region of quasi-steady energetic equilibrium. To complicate matters further, it is no longer true that the material on either side of the shock front is fully ionized. Ahead of the shock, the ionized fraction $x$ is $10 \%$ or less, while immediately behind the shock the atmosphere is fully ionized during the initial condensation evolution. As the condensation weakens propagating into the chromosphere, the ionized fraction immediately behind the shock front begins to fall short of unity, and eventually the shock causes little change in the ionized fraction. Even early on, when the condensation is moving down rapidly, the ionized fraction drops from unity just behind the shock to roughly 0.15 at the back end of the condensation, adjacent to the flare transition region. The dominant radiative loss mechanism in the thermal model condensation varies according to position: Immediately behind the shock front, Balmer continuum recombination and losses from optically thick CaII and MgII dominate, while at the back end of the condensation, Lyman $\alpha$ and Lyman continuum losses dominate. The dominant loss mechanism for the thermal model condensation therefore cannot easily be specified in an optically thin manner.

In spite of the differences between condensations in the thick target and thermal model cases, and the various complexities associated with the thermal model condensation, there are a number of simple conclusions which can be drawn from the simulations:

(1) The downflow velocity of material within the condensation is independent of column depth although it is changing with time;

(2) In both thick target and thermal model cases, the condensations 
continually slow down as they propagate into the gravitationally stratified atmosphere. This is due to two effects. One of these is inertial, i.e. the density of the material ahead of the condensation increases with depth. The other effect is that the pressure jump across the condensation front, which actually drives the downflow, is decreasing as the pressure ahead of the condensation increases with depth. A fairly good qualitative description of several of the flare simulations can be obtained by assuming that the pressure just behind the condensation front remains constant as the front propagates downward.

(3) In both heating models, the density within the condensation $\left(n_{2}\right)$ is much greater than that ahead of it $\left(n_{1}\right)$. This will remain true until the very last stages of the condensation's downward motion.

These observations allow one to develop a simple analytical model of condensation dynamics which reproduces the numerical results quite well. For example, using the preceding conclusions, it is straightforward to derive an 'equation of motion' for the condensation

$$
n^{2}=\left(n_{1} / m\right)\left(P_{2}-P_{1}\right)
$$

where $\mathbf{N}$ is the column number accretion rate of the condensation front, $n_{1}$ and $P_{1}$ are the density and pressure of the material immediately ahead of the front, and $\mathrm{P}_{2}$ is the pressure immediately behind the front, which is assumed for the moment not to vary with time. If the atmosphere ahead of the condensation is assumed to be in hydrostatic equilibrium (an excellent assumption for the thermal model, but somewhat questionable for the thick target model), described by a constant gravitational scale height $H$, then equation (21) can be integrated analytically. As a result, the time dependence of the condensation downflow velocity is found to be

$$
v_{d}(t)=\pi(H / \tau) \cot \left[(\pi / 2)\left(t / \tau+\alpha_{0}\right)\right],
$$

where $\sin ^{2}\left(\alpha_{0}\right)=N_{0} / N_{\max }$, with $N_{0} / N_{\max } \ll 1$ in general. $N_{0}$ is the initial formation depth of the condensation, $N_{\max }$ is the stopping depth $\left(\mathrm{N}_{\max }=\mathrm{P}_{2} / \mathrm{mg}\right)$, and $\tau$ is the condensation lifetime

$$
\tau=\pi(\mathrm{H} / \mathrm{g})^{1 / 2} \text {. }
$$

The actual downflow behavior for a thermal model simulation is described at least qualitatively by (22), as can be seen in Fig. (5), where the analytical result is compared to that from a simulation. The scale height $\mathrm{H}$ of 158 [ $\mathrm{km}]$ is determined by an ionized fraction of 0.1 and a chromospheric temperature of 6660 [K], consistent with the mid chromosphere of our initial atmosphere, the VERNAZZA, AVERETT, and LOESER model F [41].

The success of the above simple model in describing condensation dynamics allows the prediction of potentially observable quantities without knowing the detailed thermodynamic structure of the condensation itself. Suppose, for example, that the $H \alpha$ red wing asymmetry often observed during flares is a measure of the condensation downflow speed $v_{d}(t)$, as has been proposed by ICHIMOTO and KUROKAWA [42]. Then equation (22) predicts the temporal evolution of the velocity determined from the red wing asymetry, and that the asymmetry should die away on a time scale of $\tau$, which is about 1 minute for typical chromospheric values of the temperature and ionized fraction. Although time resolution of $\mathrm{H} \alpha$ asymmetries during flares is not sufficiently high to compare with the detailed temporal behavior of the simulations or with the analytical model, it is sufficiently good to show that the asymmetry decay time is roughly consistent with that predicted by (23). Studies are presently under way [43] to develop more realistic analytical models of condensation dynamics which relax some of the assumptions used in deriving (22), but preliminary results show that the resulting decay times are fairly close to those given by (23), and that the initial evolution of the condensation is 
still described fairly well by (22). Observational studies are also underway to study the evolution of red wing asymmetries during flares with better time resolution [44].

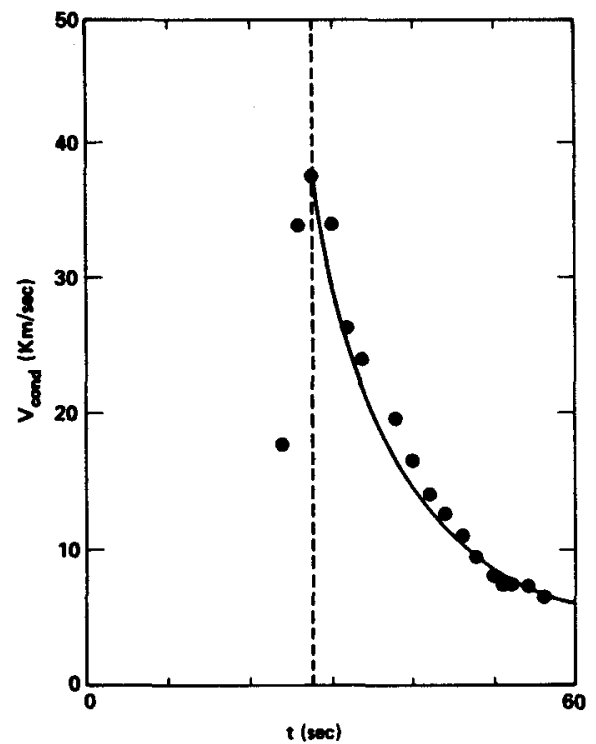

Figure 5. Comparison of
condensation downflow speed
computed in the thermal model
simulation with the result
(22). The dotted line
corresponds to t=0 [s] in
(22). The long delay before that
corresponds to the motion of the
conduction front through the
corona

VII. Radiating Shocks.

In the preceding section the precise structure of the chromospheric condensation was glossed over, as such detalled knowledge is not necessary in order to understand the overall dynamics. However, the structure of the condensation, in particular the radiating shock which may form at its leading edge, can lead to distinct emission properties which could be used as a diagnostic of the temperature and density profiles in the radiating shock, in turn yielding information on energy deposition in the flaring atmosphere. The main observational consequences of radiating shocks, if they form, is that they can provide a significant source of UV radiation in the impulsive phase of flares. In fact, as will be illustrated shortly, a radiating shock can produce much more of the emission normally associated with the transition region than does the transition region itself, at least during the transient period the radiating shock exists. To demonstrate this, the following section deals with the calculation of the differential emission measure $(E(T)$ ) in a radiating shock formed by thick target heating, and compares this with $\xi(T)$ from the transition region. In both cases, a simplified radiative loss rate is assumed.

The emission flux $F$ from an optically thin, collisionally excited process in a plasma with a non-uniform distribution of temperature and electron density can of ten be expressed in the form

$$
F=\int(g(T) / T) \xi(T) d T,
$$

where $g(T)$ absorbs the details of the atomic physics of the given emission process, and $\xi(T)$ is the differential emission measure 


$$
\xi(T)=n_{e}^{2}(d \ln T / d z)^{-1}
$$

Because of this relationship between a measurable quantity (F), a supposedly known quantity $(\mathrm{g}(\mathrm{T}))$, and the model dependent quantity $(\xi(T))$, the differential emission measure is an important contact point between theory and observation.

The structure of the transition region is of ten assumed to be determined by a balance between conductive heating and radiative losses, and as such yields the following behavior for $\xi\left(T_{5}\right)=\xi\left(T / 10^{5}[K]\right)$ :

$$
\begin{gathered}
\xi_{\mathrm{tr}}\left(\mathrm{T}_{5}\right)\left[\mathrm{cm}^{-5}\right]=1.16 \times 10^{27} \mathrm{P} \mathrm{T}_{5}{ }^{-3 / 4} \text { for } \mathrm{T}_{5}<1, \\
\xi_{\mathrm{tr}}\left(\mathrm{T}_{5}\right)\left[\mathrm{cm}^{-5}\right]=1.16 \times 10^{27} \mathrm{PT}_{5}{ }^{3 / 2}\left[1+9\left(\mathrm{~T}_{5}{ }^{1 / 2}-1\right)\right]^{-1 / 2} \text { for } \mathrm{T}_{5}>1,(26 \mathrm{~b})
\end{gathered}
$$

where $P$ is the transition region pressure [dyne $\mathrm{cm}^{-2}$ ], and the optically thin radiative loss function is assumed to have the form

$$
\begin{aligned}
& \Lambda\left(T_{5}\right)\left[\mathrm{erg} \mathrm{cm} \mathrm{c}^{-1}\right]=7 \times 10^{-22} \mathrm{~T}_{5}^{3} \text { for } T_{5}<1, \\
& \Lambda\left(T_{5}\right)\left[\mathrm{erg} \mathrm{cm} \mathrm{s}^{-1}\right]=7 \times 10^{-22} \mathrm{~T}_{5}^{-1} \text { for } T_{5}>1,
\end{aligned}
$$

which has been demonstrated to be a reasonable approximation to the actual radiative losses during thick target heating $[25,29]$. In fact, (26) is an upper limit to $\xi_{\text {tr }}(T)$, since the flare transition region is undergoing evaporation, a process which must reduce $\xi_{t r}(T)$ [45].

In a radiating shock, it is straightforward to show that $\xi(T)$ is approximately of the form

$$
\xi_{\text {rs }}(T)=5 k N T / \Lambda(T) \text {, for } T \text { cond }<T<T_{\max } \text {, }
$$

where $T_{\max }$ is determined by the strength of the shock and the temperature just ahead of $i t$, and $T_{\text {cond }}$ by the quasi-steady equilibrium condition in the bulk of the condensation. The quantity $N$ is the instantaneous column number flux through the radiating shock. By applying values of $N$ obtained from the simulations, one finds that $\xi_{r s}(T) \gg \xi_{t r}(T)$ at a given instant in time for the allowed temperature range within the radiating shock. Therefore, emission from the radiating shock will dominate that from the transition region, and should therefore be observable. There are a number of further predictions one can make. As the condensation decelerates into the gravitationally stratified chromosphere, the shock strength will weaken, reducing the peak temperature achieved just behind the hydrodynamic shock. The emission from the highest temperatures in the radiating shock will therefore 'wink out' at some early point in time, with emission from successively lower temperatures disappearing at progressively later times. This behavior, if it in fact exists, should be clearly visible by an instrument such as the (recently crippled) Ultraviolet spectrometer Polarimeter (UVSP) on board the Solar Maximum Mission.

In order to demonstrate this effect, and to show how the evolution of the radiating shock and condensation are tied together, one can combine the analytic behavior for the condensation downflow discussed in the previous section (to get an expression for $N$ ) with the expression (28) for $\xi_{\mathrm{rs}}(\mathrm{T}$ ). The condition that the atmosphere ahead of the radiating shock is in quasisteady equilibrium between flare heating and radiative losses unambiguously determines the temperature just ahead of the radiating shock. Knowing also the condensation downflow speed unambiguously determines the Mach number, and hence the maximum temperature reached in the radiating shock at each point in time. It is therefore possible to predict the entire evolution of $\xi_{r s}(T)$ over 
the life of the radiating shock. (In the case illustrated in Fig. (6), it is 21 seconds. The subsequent condensation motion (lasting $=40$ [s]) is subsonic). Three curves of $\xi(T)$ have been plotted in Fig. (6), corresponding to a specific case of thick target explosive evaporation. The dotted curve $\left(\xi_{\mathrm{rs}}(\mathrm{T})\right)$ is the differential emission measure from the radiating shock when it first forms. The dashed curve $\left(\xi_{t r}(T)\right)$ is from the transition region at the same pressure as the condensation, and the solid curve $\left(\bar{F}_{\mathrm{rs}}(\mathrm{T})\right)$ is the average from the radiating shock over the length of time the radiating shock exists. One concludes that:

(1) At high time resolution, the radiating shock emission should be clearly visible over that from the transition region at all temperatures below the shock maximum, but

(2) At low time resolution $\xi_{t r}(T)$ dominates over $\xi_{\mathrm{rs}}(\mathrm{T})$ for temperatures above $10^{5}[\mathrm{~K}]$. Nevertheless, at low temperatures (bel ow $10^{5}[\mathrm{~K}]$ ) $\xi_{\mathrm{rs}}(\mathrm{T}) \mathrm{still}$ dominates that from the transition region, and causes a much steeper temperature dependence of $\xi: \xi \mathrm{rg}(\mathrm{T}) \propto \mathrm{T}-3.0$. One concludes that $\mathrm{WV}$ instruments of roughly 10 second time resolution should be able to detect emission from the lower temperature portion of a radiating shock, and instruments with time resolution of 1 second or better should be able to detect the short lived emission from higher temperatures in radiating shocks.

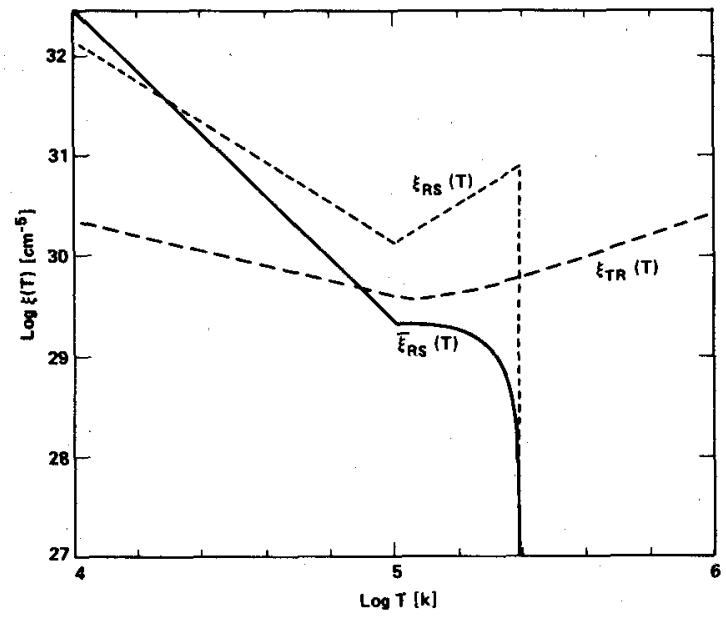

Figure 6. The dashed,
$\begin{aligned} & \text { dotted, and solid curves } \\ & \text { correspond } \\ & \text { differential }\end{aligned}$
to emission measure from the transition region, the initial state of the radiating shock, and the average over the $l$ if e of the radiating shock, respectively, for thick target heating similar to that used in Fig. 3

One significant piece of physics ignored in the entire preceding discussion is the question of ionization equilibrium. As is well known [46], the cooling time for an individual ion in a radiating shock can be shorter than its ionization equilibrium time scale, requiring a fully time dependent ionic species calculation to self-consistently determine the cooling rate in a radiating shock. Many such calculations have been done for radiating shocks in supernova remnants, for example [46], but at densities 11 or 12 orders of magnitude lower than the solar flare case. In the SNR case, important contributors to the loss rate include many forbidden and semi-forbidden transitions which would probably be quenched in the solar case. It is clear that calculations of the type performed by SHULL and MCKEE [46] need to be done at much higher densities, and need to include the rapid weakening of the shock with time in order to make realistic predictions of specific UV spectral features. However, a study of non-equilibrium ionization effects in flares undertaken by MACNEICE et al [19] showed that the actual temperature structure in such a self-consistent calculation should not differ too much from that 
reflected by the $E(T)$ calculations presented here using an ionization equilibrium radiative loss rate.

Radiating shocks in thermal model calculations seem to be significantly different from thick target radiating shocks. The chromospheric pressure enhancement due to evaporation by the conduction front in general seems to be much less than that for explosive evaporation. $[16,30]$. This results in a slower downflow speed, and hence a lower postshock temperature. In addition, the material ahead of the condensation is generally not ionized, as it has not been preheated by the nonthermal electrons. A great deal of the shock energy goes into simply ionizing the hydrogen, rather than heating the shocked material to high temperatures. As mentioned earlier, the characteristic emission in the thermal model radiating shock tends to be Balmer continuum recombination and optically thick $\mathrm{CaII}$ and $\mathrm{MgII}$ line radiation close to the shock front itself, and Lyman $\alpha$ and Lyman continuum radiation further toward the back (top) of the radiating shock. There is no natural distinction between the radiating shock and the rest of the chromospheric condensation, as there is in the thick target case.

\section{Conclusions}

The motivation for performing detailed calculations of the radiative hydrodynamic response of loop models to flare energy release is that flare observations suggest that such models might have some realistic basis. As a result of performing these calculations, however, the situation has been turned around, and it is now possible to make new predictions of potentially observable phenomena based on these calculations. For example, if the thick target model of loop heating is to be taken at face value, then one expects that:

(1) Explosive evaporation at large electron energy fluxes will initiate a downward moving chromospheric condensation, producing observable red asymmetries in $\mathrm{H \alpha}$, and the subsequent decay of these asymmetries on time scales of $T=\pi(H / g)^{1 / 2}$;

(2) Simultaneous with the initial downflow of a condensation, a bright radiating shock will form, emitting transition region-like $\mathrm{W}$ radiation, but with the higher temperature emission rapidly 'winking out' as the shock decelerates. If it is possible to measure the downflow in the condensation sufficiently accurately, one should furthermore be able to link given downflow speeds with the disappearance of specific UV transitions as the condensation slows down.

(3) The temperature dependence of the time averaged differential emission measure below $10^{5}$ [K] should be overall somewhat steeper during flares than the quiet sun, where there presumably are no radiating shocks present.

\section{Discussion:}

Frank H. Shu: You talked mostly about the condensation phase behind the radiating shock, but this must be followed by an evaporative phase in which thermal conduction eats away at the condensation. How long does this take, and is it observable?

Fisher: It is certainly true that the back end (top) of the condensation is being evaporated conductively, However, the column number accretion rate of the condensation (roughly $10^{19}$ [particles $\mathrm{cm}^{-2} \mathrm{~s}^{-1}$ ]) is much greater than the conductive evaporation rate (roughly $10^{17}$ [particles $\mathrm{cm}^{-2} \mathrm{~s}^{-1}$ ]). Basically, the condensation has already stopped by the time a significant fraction of it has been evaporated. The evaporation definitely has an observable consequence, namely the increase of the soft $x$-ray emission measure. Observed time scales for its increase are roughly consistent with those obtained with estimates of the coronal conductive flux.

Rino Bandiera: When the chromosphere is heated up, could the gas energy 
density be higher than the magnetic one?

If yes, could the magnetic field structure be affected, and in which way?

Fisher: The largest gas pressure encountered in any of the simulations of explosive evaporation was about 400 [dyne $\left.\mathrm{cm}^{-2}\right]$, corresponding to an equivalent magnetic field of about 100 [gauss]. This is not an unthinkable value, as active region loops have been observed with similar estimated field strengths. In such a case, I imagine that the evaporated region might initially bulge out, resulting in Alfven waves driven along the loop. In addition, magnetoacoustic waves might be driven out in a perpendicular direction into the adjacent flux tubes. The dissipation of the energy in these ways would reduce that available for mass flow along the loop, and the upflow speeds would probably be reduced. However, there is some evidence that compact flare loops have much higher field strengths than this, in which case the rigid tube approach is still a good approximation.

Vincent Icke: I'm worried about your geometry. In double ribbon flares there's lots of evidence that the magnetic configuration changes rapidly, so that new material is being bombarded all the time. Does that not require changes in your approach?

Fisher: I presume you're referring to the expansion of the Ha ribbons, and the interpretation of that in the Kopp and Pneumann reconnection picture, where new loops are continuously forming through the reconnection of open field lines. In that case, I would expect that the simple closed flux tube model I discussed here would work once a closed flux tube had been formed, but would be irrelevant before that. One would then need to somehow superimpose a collection of these 1-d loop models in order to describe the evolving arcade.

Vincent Icke: Would you agree that the radio observations favor the particle injection (thick target) model over the thermal model?

Fisher: Yes. During the impulsive phase, the observation of what is apparently gyrosynchrotron emission in the microwave spectrum, and type III radio bursts, apparently due to beams of electrons accelerated into the interplanetary medium, argue strongly in favor of particle acceleration.

Reuven Opher: The damping rate of turbulent Alfven waves is proportional to $\nu k^{2} / n$, where $v$ is the collision frequency, $n$ is the number density, and $k$ is the average wave number determined by the turbuient spectrum which can be fairly large. Appreciable absorption can occur in the corona and in the transition region. How do these waves affect your model and what are the observable consequences?

Fisher: The absorption of Alfven waves has been proposed as a heating mechanism in flares by Emslie and Sturrock. They were most interested in exploring the possibility that the observed heating of the temperature minimum region might be explained by Alfven waves. Emslie now says he believes that al though this is possible, one must pick a contrived set of parameters for it to work properly.

Reuven Opher: The proposed mechanism for producing the fast electrons is magnetic reconnection which is occuring in a highly turbulent region. This region should also produce a large fraction, or a major fraction, of its energy in turbulent Alfven waves which will propagate at the Alfven velocity. For a typical loop dimension they should reach the transition region on the order of a second, which is in agreement with the Oxygen $V$ line data.

Miguel H. Ibanez S.: Have you checked whether thermal and magneto-acoustic waves play some role in your models? 
Fisher: A self-consistent treatment of magneto-acoustic waves in these 1-d models has essentially been precluded by assuming that the loop is a rigid tube. One can include the effects in an ad-hoc manner by specifying (for example) what heating effects one expects the waves to have, but this has not been done in these models. As $f$ ar as thermal waves in 1-d loop models goes, a considerable amount of effort has gone into the study of the stability of thermal waves in solar loops. You might want to look at the work by Antiochos and by McClymont and Craig.

Ake Nordlund: I don't want to be negative over a good talk, and good work, but the statement that "The probabilistic radiative transfer has been tested and has been found to do quite a good job" would need some additional comments. I am skeptical about P.R.T. in situations with super Doppler velocity fields.

Fisher: You're quite right in being skeptical in the case of velocity gradients in optically thick material. We employ escape probabilities that are computed using the static atmosphere assumption. The validity of this is tested in the numerical code using criteria formulated by Hummer and Rybicki, and we find that in nearly all cases $d v / d \tau$ in hydrogen transitions is small enough that the static assumption is OK. However, I expect that the escape probabilities in use for $\mathrm{CaII}$ and $\mathrm{MgII}$ transitions are probably too low, for this reason. That means the CaII and MgII losses are probably underestimated in the region just behind the radiating shock,' for example.

Dmitri Minalas: How important is escape of radiation out the sides of your loop model as compared to escape out the top of the chromosphere?

Fisher: Well, that depends on the ratio of the width of the chromospheric portion of a compact flare loop to its height, which I don't think is terribly well known. My guess, from the size of bright Ha kernels during the impulsive phase, is that the width of such loops is roughly the same as the chromospheric depth, namely 1000 or $2000 \mathrm{~km}$. It probably varies from loop to loop. This effect is not included in the numerical models.

John Brown: Does the creation of condensations, and the existence of a threshold for explosive evaporation, depend on the instantaneous switch-on of your beam? Would they vanish if the beam were ramped up gradually?

Fisher: Given an average heating rate (per particle) $Q_{0}$, a column thickness of the chromosphere $N_{0}$ unable to radiate away the flare heating, with the spatial extent of this region being $L_{0}$, then the timescale necessary to achieve explosive evaporation $\left(\tau_{\text {exp }}\right)$ is $\tau_{\exp }=\left[\mathrm{mL}_{0} 2 /\left(2 Q_{0}\right)\right]^{1 / 3}$, where $\mathrm{m}$ is the mean mass per hydrogen nucleus. If $\tau_{\text {exp }} \geqslant \tau_{r i s e}$, where $\tau_{r i s e}$ is the rise time for the beam energy flux, then I expect explosive evaporation to occur, and to initiate the formation of a condensation. It should be possible, with spatially and temporally resolved hard $\mathrm{x}$-ray observations, to estimate both of these time scales. If one can indeed detect condensations through $H_{\alpha}$ asymmetries, then it should be possible to make a consistency check of this whole scenario by seeing if the existence of red wing asymmetries depends on the above time scale inequality.

John Brown: Regarding the numerical techniques, could you summarize the current state of the SMM hydrodynamic 'benchmark' exercise in which there were serious discrepencies between different codes run for the same problem?

Fisher: There were indeed serious discrepencies between the different codes. It turned out some of these were due to what seemed initially to be minor details such as using different assumptions about the ambient heating function used to keep the initial atmosphere in energetic equilibrium. other discrepencies might be explained by insufficient numerical resolution of the transition region in some of the models. 
John Brown: In response to the question by Rino Bandiera: It has been suggested by A. G. Emslie that energy deposition by the beam could indeed produce a gas pressure exceeding the magnetic pressure, and that the resulting flux tube expansion could act as a trigger on neighboring tubes and initiate subsequent further reconnection and acceleration.

In response to the question by Reuven Opher on Alfven waves, Emslie and Sturrock studied Alfven wave heating. While it is possible, for carefully chosen parameters, to heat the chromosphere in this way, the process is too slow (for the observations) at layers much below the transition region because the Alfven speed becomes very low there.

Two final comments I would make, of possibly more general interest here are:

(1) This paper is the first mention we have had of nonthermal particles as an important element in the hydrodynamic equations. They may also be relevant in accretion columns and around QSO's.

(2) If the flux tube is not uniform in cross-section, there arises the important effect of loss-cone instability and generation of radiation by masering (Melrose and Dulk). Such radiation (decimetric for solar parameters) plays the role of transporting energy to (and hence driving motions in) the plasma outside the tube, hence turning a 1-d problem into a 3-d problem. This process may also have analogues in cosmic ray problems.

\section{References:}

1. Krieger, A.S.: "X-ray Observations of Solar Structural Features", in Proceedings of the OSO-8 Workshop, (Laboratory for A tmospheric and Space Sciences, University of Colorado 1977)

2. Svestka, Z.: Solar Flares, (D. Reidel Publ. Co., Dordrecht, Holland (1976)

3. Pneumann, G.W.: "Two Ribbon Flares: (post) Flare Loops", in Solar Flare Magnetohydrodynamics, E.R. Priest (ed), (Gordon and Breach Publ., New York 1981)

4. Kopp, R.A., Pneumann, G.W.: Solar Physics 50, 85. (1976)

5. Woodgate, B.E., Shine, R.A., Poland, A.I., Orwig, L.E.: Astrophysical Journal 265, 530. (1983)

6. Canfield, R.C., Gunkler, T.A.: Astrophysical Journal 288, 353. (1985)

7. Kane, S.R., Donnelly, R.F.: Astrophysical Journal 164, 151. (1971)

8. Antonucci, E.,Gabriel, A.H., Dennis, B.R.: Astrophysical Journal 287 , 917. (1984)

9. Brown, J.C.: Solar Physics 26, 441. (1972)

10. Lin, R.P., Hudson, H.S.: Solar Physics 50, 153. (1976)

11. Kostyuk, N.D., Pikel'ner, S.B.: Soviet Astronomy, 18, 590. (1975)

12. Kostyuk, N.D.: Soviet Astronomy, 20, 206. (1976)

13. Craig, I.J.D., MeClymont, A.N.: Solar Physies 50, 133. (1976)

14. Livshitz, M.A., Badalyan, O.G., Kosovichev, A.G., Katsova, M.M.: Solar Physics 73, 269. (1981)

15. Somov, B.V., Syrovatskii, S.I., Spektor, A.R.: Solar Physics 73, 145. 
(1981)

16. Cheng, C.C., Oran, E.S., Doschek, G.A., Boris, J.P., Mariska, J.T.: Astrophysical Journal 265, 1090. (1983)

17. Pallavicini, R., Peres, G., Serio, S., Vaiana, G., Acton, L., Leibacher, J., Rosner, R.: Astrophysical Journal 270, 270. (1983)

18. Duijueman, A., Somov, B.V., Spektor, A.R.: Solar Physics 88, 257. (1983)

19. MacNeice, P., McWhirter, R.W.P., Spicer, D.S., Burgess, A.: Solar Physics 90, 357. (1984)

20. Cheng, C.C., Karpen, J.T., Doschek, G.A.: Astrophysical Journal 286, 787. (1984)

21. Machado, M.E., Averett, E.H., Vernazza, J.E., Noyes, R.W.: Astrophysical Journal 242, 336. (1981)

22. Ricchiazzi, P.J., Canfield, R.C.: Astrophysical Journal 272, 739. (1983)

23. McClymont, A.N., Canfield, R.C.: Astrophysical Journal 265, 483 (1983)

24. Canfield, R.C., Fisher, G.H., MCClymont, A.N.: Astrophysical Journal 289, 507. (1983)

25. McClymont, A.N., Canfield, R.C.: Astrophysical Journal 265, 497. (1983)

26. An, C.H., Canfield, R.C., Fisher, G.H., McClymont, A.N.: Astrophysical Journal 267, 421. (1983)

27. Fisher, G.H., Canfield, R.C., MoClymont, A.N.: Astrophysical Journal 289, 414. (1985)

28. Fisher, G.H., Canfield, R.C., McClymont, A.N.: Astrophysical Journal 289, 425. (1985)

29. Fisher, G.H., Canfield, R.C., McClymont, A.N.: Astrophysical Journal 289, 434. (1985)

30. Fisher, G.H.: In prep. (1985)

31. Canfield, R.C., McClymont, A.N., Puetter, R.C.: "Probabilistic Radiative Transfer", in Methods in Radiative Transfer, ed. Wolf gang Kal kof en (Cambridge Univ. Press, 1984)

32. Henoux, J.C., Nakagawa, Y, : Astronomy and Astrophysies 57, 105. (1977)

33. Ricchiazzi, P.J.: PhD Thesis, University of California at San Diego (1982)

34. Raymond, J.C., Cox, D.P., Smith, B.W.: Astrophysical Journal 204. 290. (1976)

35. Raymond, J.C.: Private communication to P. J. Ricchiazzi (1980)

36. Spitzer, L.: Physics of Fully Ionized Gases (Interscience, New York 1962)

37. Hauer, A., Mead, W.C., Willi, O., Kilkenny, J.D., Bradley, D.K., Tabatabaei, S.D., Hooker, C.: Physlcal Review Letters, 53, 2563. (1984)

38. Luciani, J.F., Mora, P., Pallat, R.: Physics of Fluids, 28, 835. (1985) 
39. Karpen, J.T., Devore, C.R.: Astrophysical Journal, submitted. (1985)

40. Fisher, G.H., Canfield, R.C., McClymont, A.N.: Astrophysical Journal (Letters) 281, L79 (1984)

41. Vernazza, J.E., Averett, E.H., Loeser, R.: Astrophysical Journal (Supplement) 45, 619. (1981)

42. Ichimoto, K., Kurokawa, H.: Solar Physics 93, 105. (1984)

43. Fisher, G.H.: In prep. (1985)

44. Canfield, R.C.: Private communication. (1985)

45. Craig, I.J.D., McClymont, A.N.: Astrophysical Journal (submitted) (1985)

46. Shull, J.M., McKee, C.F.: Astrophysical Journal 227, 131 (1979) 